\title{
ON SQUARE-FULL INTEGERS IN A SHORT INTERVAL
}

\author{
by P. SHIU.
}

(Received 26 November, 1982)

1. Introduction. A positive integer $n$ is called a square-full integer if $p^{2}$ divides $n$ whenever $p$ is a prime divisor of $n$. For $x \geqslant 1$ we denote by $Q(x)$ the number of square-full integers not exceeding $x$. Bateman and Grosswald [1] proved that

$$
Q(x)=A x^{1 / 2}+B x^{1 / 3}+\Delta(x)
$$

where

and

$$
A=\frac{\zeta\left(\frac{3}{2}\right)}{\zeta(3)}, \quad B=\frac{\zeta\left(\frac{2}{3}\right)}{\zeta(2)}
$$

$$
\Delta(x)=o\left(x^{1 / 6}\right) \text { as } x \rightarrow \infty .
$$

They also pointed out that the exponent $\frac{1}{6}$ here can be reduced if and only if the supremum of the real parts of the zeros of $\zeta(s)$ is less than 1 .

We shall be concerned with the number of square-full integers in the interval $x<n \leqslant x+h$. It is proved in [8] that there may be no square-full integer between successive squares so that if $h<\sqrt{x}$, then the interval $x<n \leqslant x+h$ may contain no square-full integer at all. We shall write

$$
h=x^{1 / 2+\theta} \quad\left(0<\theta<\frac{1}{2}\right) .
$$

It follows at once from the result of Bateman and Grosswald that if $\frac{1}{6}<\theta<\frac{1}{2}$, then

$$
Q(x+h)-Q(x) \sim \frac{1}{2} A x^{\theta} \quad \text { as } \quad x \rightarrow \infty .
$$

The purpose of this paper is to extend this interval result to the shorter range

$$
0 \cdot 1526 \leqslant \theta \leqslant \frac{1}{6} \text {. }
$$

The method employed is that introduced by Roth [7] who obtained a short interval result for square-free integers. The method reduces the problem to that of the estimation of the two dimensional exponential sum given in (3.11). We shall also require an estimation of the error term associated with the asymptotic formula for the sum

$$
S(x)=\sum_{a^{2} b^{3} \leqslant x} 1
$$

2. The asymptotic formula for $S(x)$. Let $\psi(x)=x-[x]-\frac{1}{2}$ and, for positive $\alpha, \beta, \gamma$, write

$$
R(x ; \alpha, \beta, \gamma)=\sum_{n \leq x^{\alpha}} \psi\left(\frac{x^{\beta}}{n^{\gamma}}\right)
$$

Glasgow Math. J. 25 (1984) 127-134. 
Richert [6] showed that, for distinct positive constants $u$ and $v$, the sum $\sum_{a^{u} b^{v} \leqslant x} 1$ has an asymptotic formula whose error term can be expressed in terms of $R(x ; \alpha, \beta, \gamma)$ with $\alpha, \beta, \gamma$ depending on $u$ and $v$. In particular we have, for the sum in (1.7),

$$
S(x)=\zeta\left(\frac{3}{2}\right) x^{1 / 2}+\zeta\left(\frac{2}{3}\right) x^{1 / 3}+\Delta^{*}(x),
$$

where

$$
\Delta^{*}(x)=-R\left(x ; \frac{1}{5}, \frac{1}{2}, \frac{3}{2}\right)-R\left(x ; \frac{1}{5}, \frac{1}{3}, \frac{2}{3}\right)+O(1) .
$$

It follows trivially from (2.1) that $\Delta^{*}(x) \ll x^{1 / 5}$ as $x \rightarrow \infty$. We define $\rho^{*}$ to be the infimum of the set of real numbers $\rho$ such that $\Delta^{*}(x) \ll x^{\rho}$ holds as $x \rightarrow \infty$. From an application of a special case of the colossal lattice point theorem due to Landau [3] we see that

$$
\rho^{*} \geqslant \frac{1}{10} .
$$

We use the method of exponent pairs to prove that

$$
\rho^{*} \leqslant 0 \cdot 13181619 \ldots
$$

Van der Corput's method of estimating trigonometric sums has been developed into a delicate theory of exponent pairs due to van der Corput [10], Phillips [4], and Rankin [5]. The rather complicated definition of an exponent pair is given in [4] and [5]. By means of this theory Richert [6] has proved the following result.

Lemma 1. (Richert [6, Lemma 8]) Let $\alpha, \beta, \gamma$ be positive constants and let $(k, l)$ be an exponent pair. Then, as $x \rightarrow \infty$,

$$
R(x ; \alpha, \beta, \gamma) \ll x^{\alpha-\frac{1}{2}(\beta-\alpha \gamma)}+ \begin{cases}x^{(\alpha l+(\beta-\alpha \gamma) k) /(k+1)} & \text { if } l>\gamma k, \\ x^{\beta k /(k+1)} \log x & \text { if } l=\gamma k, \\ x^{\beta k /(1+(\gamma+1) k-1)} & \text { if } l<\gamma k .\end{cases}
$$

Let $(k, l)$ be an exponent pair such that $2 l=3 k$. From Lemma 1 and $(2.3)$ we see that, as $x \rightarrow \infty$,

$$
\Delta^{*}(x) \ll x^{k /(2 k+2)} \log x
$$

since, by the definition of an exponent pair, we must have $l \geqslant \frac{1}{2}$ so that $k \geqslant \frac{1}{3}$ giving $k /(2 k+2) \geqslant \frac{1}{8}>\frac{1}{10}$. Therefore, for $2 l=3 k$, we have

$$
\rho^{*} \leqslant \frac{k}{2 k+2} \text {. }
$$

Next, let $\eta$ be any positive number such that $\left(\eta, \frac{1}{2}+\eta\right)$ is an exponent pair. By applying the $A$ and $B$ processes of Phillips [4], namely $B A\left(\eta, \frac{1}{2}+\eta\right)$ and $B A^{2}\left(\eta, \frac{1}{2}+\eta\right)$, and then the convexity process of Rankin [5] we see that

$$
\left(\frac{2 \eta+1}{2 \eta^{2}+4 \eta+3}, \quad \frac{3}{2} \times \frac{2 \eta+1}{2 \eta^{2}+4 \eta+3}\right)
$$


is now an exponent pair $(k, l)$ satisfying $2 l=3 k$. Consequently we have

$$
\rho^{*} \leqslant \frac{2 \eta+1}{4(\eta+1)(\eta+2)}
$$

Moreover, Rankin [5] showed that the infimum of the set of $\eta$ such that $\left(\eta, \frac{1}{2}+\eta\right)$ is an exponent pair is given by

$$
\eta_{0}=0 \cdot 1645106784 \ldots
$$

so that (2.5) now follows from (2.6).

3. Roth's method. We shall prove the following result.

THEOREM. Let $\theta_{0}$ be the infimum of the set of all numbers $\theta$ such that (1.5) holds. Then

$$
\theta_{0} \leqslant \frac{1+\rho^{*}}{9-12 \rho^{*}}
$$

We remark that the right hand side of (3.1) is less than $\frac{1}{6}$ if and only if $\rho^{*}<\frac{1}{6}$; and that the asymptotic formula (1.5) holds when $\theta$ lies in the interval (1.6) follows from the theorem together with the estimate (2.5).

We now apply Roth's method [7] to investigate $Q(x+h)-Q(x)$. Let $\rho$ and $\theta$ satisfy

$$
\rho *<\rho<\frac{1}{6}, \quad 0<\theta<\frac{1}{2}
$$

and, for $x>1$, we let

$$
h=x^{1 / 2+\theta}, \quad \log x<t<x^{1 / 6} .
$$

Since a square-full integer $q$ can be written uniquely as $a^{2} b^{3}$, where $a, b$ are positive integers and $b$ is square-free, it follows that

$$
\begin{aligned}
Q(x) & =\sum_{q \leqslant x} \sum_{a^{2} b^{3}=q} \mu^{2}(b)=\sum_{q \leqslant x} \sum_{a^{2} b^{3}=q} \sum_{c^{2} d=b} \mu(c) \\
& =\sum_{a^{2} d^{3} c^{6} \leqslant x} \mu(c) .
\end{aligned}
$$

Consequently we have

$$
Q(x+h)-Q(x)=\sum_{x<a^{2} d^{3} c^{6} \leq x+h} \mu(c)=\Sigma_{1}+\Sigma_{2}
$$

say, where

$$
\Sigma_{1}=\sum_{\substack{x<a^{2} d^{3} c^{6} \leq x+h \\ c \leqslant 1}} \mu(c), \quad \Sigma_{2}=\sum_{\substack{x<a^{2} d^{3} c^{6} \leqslant x+h \\ c>t}} \mu(c)
$$


First we have

$$
\begin{aligned}
\Sigma_{1} & =\sum_{c \leqslant t} \mu(c) \sum_{x c^{-6}<a^{2} b^{3} \leqslant(x+h) c^{-6}} 1 \\
& =\sum_{c \leqslant t} \mu(c)\left\{S\left(\frac{x+h}{c^{6}}\right)-S\left(\frac{x}{c^{6}}\right)\right\} .
\end{aligned}
$$

Now, as $x \rightarrow \infty$,

and

$$
\begin{gathered}
(x+h)^{1 / 2}-x^{1 / 2}=\frac{1}{2} x^{\theta}+O\left(x^{2 \theta-1 / 2}\right), \\
(x+h)^{1 / 3}-x^{1 / 3} \ll x^{\theta-1 / 6},
\end{gathered}
$$

$$
\sum_{c \leqslant t}\left(\frac{x}{c^{6}}\right)^{\rho} \ll x^{\rho} t^{1-6 \rho}
$$

so that, from (1.2) and (2.2), we have

$$
\Sigma_{1}=\left(\frac{1}{2} A+o(1)\right) x^{\theta}+O\left(x^{\rho} t^{1-6 \rho}\right) .
$$

Next we put

so that

$$
T(x, t)=\sum_{\substack{a^{2} b^{3} c^{6} \leq x \\ c>1}} 1
$$

$$
\left|\Sigma_{2}\right| \leqslant T(x+h, t)-T(x, t)
$$

and it now follows from (3.4) and (3.7) that

$$
\left|Q(x+h)-Q(x)-\left(\frac{1}{2} A+o(1)\right) x^{\theta}\right| \leqslant T(x+h)-T(x, t)+O\left(x^{\rho} t^{1-6 \rho}\right) .
$$

In order to estimate $T(x, t)$ we define

$$
\tau(n)=\sum_{a^{2} b^{3}=n} 1
$$

so that, by (3.8),

$$
\begin{aligned}
T(x, t) & =\sum_{\substack{n c^{6} \leqslant x \\
c>t}} \tau(n)=\sum_{n \leqslant x t^{-6}} \tau(n) \sum_{t<c \leqslant(x / n)^{1 / 6}} 1 \\
& =\sum_{n \leqslant x t^{-6}} \tau(n)\left\{\left[\left(\frac{x}{n}\right)^{1 / 6}\right]-[t]\right\} \\
& =\sum_{n \leqslant x t^{-6}} \tau(n)\left\{\left(\frac{x}{n}\right)^{1 / 6}-\psi\left(\left(\frac{x}{n}\right)^{1 / 6}\right)\right\}-t S\left(x t^{-6}\right),
\end{aligned}
$$

provided that $\psi(t)=0$, and this we may assume by taking $t$ to be half an odd integer. Now, by $(2.2)$,

$$
t S\left(x t^{-6}\right)=\zeta\left(\frac{3}{2}\right) x^{1 / 2} t^{-2}+\zeta\left(\frac{2}{3}\right) x^{1 / 3} t^{-1}+t \Delta^{*}\left(x t^{-6}\right)
$$


and, similarly to the derivation of (2.2) itself, we have

$$
\sum_{n \leq x t^{-6}} \tau(n)\left(\frac{x}{n}\right)^{1 / 6}=\frac{3}{2} \zeta\left(\frac{3}{2}\right) x^{1 / 2} t^{-2}+2 \zeta\left(\frac{2}{3}\right) x^{1 / 3} t^{-1}+\zeta\left(\frac{1}{3}\right) \zeta\left(\frac{1}{2}\right) x^{1 / 6}+t \Delta^{*}\left(x t^{-6}\right) .
$$

Let us write

$$
U(x, t)=\sum_{n \leqslant x t^{-6}} \tau(n) \psi\left(\left(\frac{x}{n}\right)^{1 / 6}\right)=\sum_{a^{2} b^{3} \leqslant x t^{-6}} \psi\left(\left(\frac{x}{a^{2} b^{3}}\right)^{1 / 6}\right) .
$$

In view of (3.5) and (3.6) it now follows from (3.10) that

$$
T(x+h, t)-T(x, t)=-U(x+h, t)+U(x, t)+O\left(x^{\rho} t^{1-6 \rho}\right)+o\left(x^{\theta}\right)
$$

and so, from (3.9), we have

$$
\left|Q(x+h)-Q(x)-\left(\frac{1}{2} A+o(1)\right) x^{\theta}\right| \leqslant|U(x+h, t)-U(x, t)|+O\left(x^{\rho} t^{1-6 \rho}\right) .
$$

The two dimensional exponential sum $U(x, t)$ in (3.11) has the trivial estimate

$$
|U(x, t)| \leqslant \sum_{a^{2} b^{3} \leqslant x t^{-6}} 1=S\left(x t^{-6}\right) \ll x^{1 / 2} t^{-3} .
$$

However, for our sharper result we need the following lemma.

LEMMA 2. As $x \rightarrow \infty$, we have

$$
U(x, t) \ll x^{1 / 2} t^{-7 / 2} \log ^{2} x
$$

uniformly for $\log x<t \leqslant x^{\beta}$, where

$$
\beta=\frac{421}{3841}=0 \cdot 1096 \ldots
$$

We give the proof of Lemma 2 in the next section. The estimate (3.14) allows us to put

$$
t=x^{(1-2 \rho) /(9-12 \rho)}
$$

since the exponent for $x$ here is at most $\frac{4}{39}<\beta$, because $\rho>\rho^{*} \geqslant \frac{1}{10}$ by (3.2) and (2.4). This choice of $t$ now gives

$$
x^{1 / 2} t^{-7 / 2}=x^{\rho} t^{1-6 \rho}=x^{(1+\rho) /(9-12 \rho)}
$$

so that from (3.12) and (3.14) we have

$$
Q(x+h)-Q(x)=\left(\frac{1}{2} A+o(1)\right) x^{\theta}+O\left(x^{(1+\rho) /(9-12 p)} \log ^{2} x\right) .
$$

Consequently if $\theta$ satisfies

$$
\frac{1+\rho}{9-12 \rho}<\theta<\frac{1}{2}
$$

then the asymptotic formula (1.5) holds. Therefore the required result (3.1) is established subject to the proof of Lemma 2. 
4. The estimation of $U(x, t)$. We write

$$
\begin{aligned}
& U_{1}(x, t)=\sum_{\substack{m^{2} n^{3} \leq x t^{-6} \\
m>n}} \psi\left(\left(\frac{x}{m^{2} n^{3}}\right)^{1 / 6}\right), \\
& U_{2}(x, t)=\sum_{\substack{m^{2} n^{3} \leq x t^{-6} \\
m<n}} \psi\left(\left(\frac{x}{m^{2} n^{3}}\right)^{1 / 6}\right),
\end{aligned}
$$

so that from (3.11) we have

$$
U(x, t)=U_{1}(x, t)+U_{2}(x, t)+O\left(\left(x t^{-6}\right)^{1 / 5}\right) .
$$

Van der Corput's method of estimating trigonometric sums has been extended to higher dimensions. In particular there is now a theory of two dimensional exponent pairs. We shall apply the following useful result due to Srinivasan [9, Main theorem].

Lemma 3. Let $\rho, \sigma>0$ and $\left(\lambda_{0}, \lambda_{1}\right)$ be any two dimensional exponent pair. Let $z, M, N$, F satisfy

$$
F=z M^{-\rho} N^{-\sigma}, \quad 1 \ll M \ll F, \quad 1 \ll N \ll F .
$$

Then, for any region $D$ in the rectangle $M<m \leqslant 2 M, N<n \leqslant 2 N$, we have

$$
\sum_{(m, n) \in D} \psi\left(\frac{z}{m^{\rho} n^{\sigma}}\right) \ll\left\{F^{1 / 2+\lambda_{0}-\lambda_{1}} M^{1 / 2+2 \lambda_{0}} N^{3 / 2-2 \lambda_{1}}\right\}^{1 /\left((3 / 2)+\lambda_{0} \lambda\right)}+F^{1 / 4} M^{1 / 4} N+F^{-1 / 2} M N
$$

The definition of a two dimensional exponent pair is given in [9] where it is also shown that $\left(\frac{23}{250}, \frac{45}{450}\right)$ is such a pair. We now apply Lemma 3 to estimate $U_{1}(x, t)$ and $U_{2}(x, t)$.

Let $z=x^{1 / 6}$ and $(\rho, \sigma)$ be either $\left(\frac{1}{2}, \frac{1}{3}\right)$ or $\left(\frac{1}{3}, \frac{1}{2}\right)$, and put

$$
S_{\rho, \sigma}(z, t ; M, N)=\sum_{\substack{M<m \leqslant 2 M \\ N<n \leqslant 2 N \\ m^{\circ} n^{\sigma} \leq z t^{-1} \\ n>n}} \psi\left(\frac{z}{m^{\rho} n^{\sigma}}\right) .
$$

Using the exponent pair $\left(\frac{23}{250}, \frac{45}{250}\right)$, Lemma 3 yields

$$
S_{\rho, \sigma}(z, t ; M, N) \ll\left(F^{92} M^{171} N^{263}\right)^{1 / 342}+F^{1 / 4} M^{1 / 4} N+F^{-1 / 2} M N,
$$

where

$$
F=z M^{-\rho} N^{-\rho}
$$

From

$$
N^{5 / 6} \ll M^{1 / 3} N^{1 / 2} \ll M^{\rho} N^{\sigma} \ll z t^{-1}
$$

it is easy to verify that

$$
\begin{gathered}
\left(F^{92} M^{171} N^{263}\right)^{1 / 342} \ll x^{217 / 855} t^{-1072 / 855}, \\
F^{1 / 4} M^{1 / 4} N \ll x^{1 / 4} t^{-5 / 4}
\end{gathered}
$$


and

$$
F^{-1 / 2} M N \ll x^{1 / 2} t^{-7 / 2}
$$

Since

$$
x^{1 / 4} t^{-5 / 4} \leqslant x^{217 / 855} t^{-1072 / 855}
$$

if and only if $t \leqslant x$, it follows that

$$
S_{\rho, \sigma}(z, t ; M, N) \ll x^{1 / 2} t^{-7 / 2}+x^{217 / 855} t^{-1072 / 855}
$$

and therefore, as $x \rightarrow \infty$,

$$
U_{1}(x, t)+U_{2}(x, t) \ll\left\{X^{1 / 2} t^{-7 / 2}+x^{217 / 855} t^{-1072 / 855}\right\} \log ^{2} x
$$

uniformly in $1 \leqslant t \leqslant x^{1 / 6}$. Since the first term on the right hand side of (4.2) dominates over the second term when $t \leqslant x^{\beta}, \beta=\frac{421}{3841}$ we see that Lemma 2 follows from (4.1) and (4.2).

\section{Remarks.}

(1) It may be of interest to point out that the simple combinatorial argument suggested by Estermann in Roth's paper also applies here to give an estimate for $T(x+h, t)-T(x, t)$ which is needed in (3.9). Let $u$ be a number satisfying $u t^{5}>h$. Corresponding to each pair of numbers $a, b$ there are at most $u$ numbers $c$ satisfying $c>t$ and $x<a^{2} b^{3} c^{6} \leqslant x+h$ since

$$
a^{2} b^{3}(c+u)^{6}>a^{2} b^{3} c^{6}+u c^{5}>x+u t^{5}>x+h .
$$

From (3.8) we see therefore that

$$
T(x+h, t)-T(x, t) \leqslant \sum_{a^{2} b^{3} \leqslant 2 x t^{-6}} u \ll u x^{1 / 2} t^{-3} .
$$

On setting $t=x^{1 / 8} \log x, u=x^{\theta-1 / 8}$ we can deduce from (3.9) that $\theta_{0} \leqslant\left(1+2 \rho^{*}\right) / 8$. We note that this result is inferior to $\theta_{0} \leqslant 1 /\left(8-12 \rho^{*}\right)$ which can be derived from (3.12) together with the trivial estimate (3.13) for the sum $U(x, t)$. That is we have

$$
\frac{1+\rho^{*}}{9-12 \rho^{*}} \leqslant \frac{1}{8-12 \rho^{*}} \leqslant \frac{1+2 \rho^{*}}{8} \leqslant \frac{1}{6}
$$

and indeed with strict inequality since we know that $\rho^{*}<\frac{1}{6}$.

(2) The distribution of $k$-full integers has been investigated recently in [2]. For example the asymptotic formula for $Q_{3}(x)$, the number of cube-full integers not exceeding $x$, has an error term $\Delta_{3}(x)$ and is related to the asymptotic formula for the sum

$$
S_{3}(x)=\sum_{a^{3} b^{4} c^{5} \leq x} 1
$$

which has an error term $\Delta_{3}^{*}(x)$. Corresponding to $\Delta_{3}(x)$ and $\Delta_{3}^{*}(x)$ let $\rho_{3}$ and $\rho_{3}^{*}$ be defined similarly to $\rho^{*}$. The upper estimates given in [2] for $\rho_{3}$ and $\rho_{3}^{*}$ are both the same, namely

$$
\frac{263}{2052}=0 \cdot 128 \ldots
$$


If it can be proved that $\rho_{3}^{*}<\frac{1}{8}$, then our short interval result here can be extended to cube-full integers.

\section{REFERENCES}

1. P. T. Bateman and E. Grosswald, On a theorem of Erdös and Szekeres, Illinois J. Math. 2 (1958), 88-98.

2. A. Ivic and P. Shiu, The distribution of powerful integers, Illinois J. Math. 26 (1982), $576-590$.

3. E. Landau, Über die Anzahl der Gitterpunkte in gewissen Bereichen (II), Nachr. Ges. Wiss. Göttingen (1915), 209-243.

4. E. Phillips, The zeta-function of Riemann: further developments of van der Corput's method, Quart. J. Math. Oxford 4 (1933), 209-225.

5. R. A. Rankin, van der Corput's method and the theory of exponent pairs, Quart. J. Math. Oxford Ser. 26 (1955), 147-153.

6. H. E. Richert, Über die Anzahl Abelscher Gruppen gegebener Ordnung (I), Math. Z. 56 (1952), 21-32.

7. K. F. Roth, On the gaps between squarefree numbers, J. London Math. Soc. 26 (1951), 263-268.

8. P. Shiu, On the number of square-full integers between súccessive squares, Mathematika 27 (1980), 171-178. 195-205.

9. B. V. Srinivasan, On the number of Abelian groups of a given order, Acta Arith. 23 (1973),

10. J. G. van der Corput, Verschärfung der Abschätzung beim Teilerproblem, Math. Ann. 87 (1922), 39-65.

Department of Mathematics

UNIVERSITY OF TECHNOLOGY

LOUGHBOROUGH

LEICESTERSHIRE

ENGLAND 